\title{
MICA - A new generation of versatile instruments in robotic surgery
}

\author{
Sophie Thielmann, Ulrich Seibold, Robert Haslinger, Georg Passig, Thomas Bahls, Stefan Jörg, \\ Mathias Nickl, Alexander Nothhelfer, Ulrich Hagn and Gerd Hirzinger*
}

\begin{abstract}
Robotic surgery systems are highly complex and expensive pieces of equipment. Demands for lower cost of care can be met if these systems are employable in a flexible manner for a large variety of procedures. To protect the initial investment the capabilities of a robotic system need to be expandable as new tasks arise.

To oblige the needs of future robotic support in hospitals, the German Aerospace Center (DLR) has developed the versatile robotic system MiroSurge for medical applications.

This paper presents a $3 \mathrm{DoF}$ instrument for Minimally Invasive Robotic Surgery which is mounted to the hollow wrist of the DLR MIRO robot arm. The MICA instrument consists of a versatile drive train and a detachable task specific tool with its tool interface, shaft, 2 DoF wrist, 7 DoF force/torque sensor and the actuated functional end. With the current cabledriven tool, gripping and manipulation forces of above $10 \mathrm{~N}$ are feasible and dynamics is high enough for surgery at the beating heart.
\end{abstract}

\section{INTRODUCTION}

Versatility in robotic surgery is a step towards making medical robotics more affordable. Versatile robots can be used in a wide range of medical applications, from endoscopic telesurgery, to guidance of endoscopes, assistance in laser osteotomies, detection of chest wall arteries, placement of pedicle screws and more. With a versatile robot the dedication to a specific application area is accomplished by programming and by changing the specific instrument, not the robot arm.

Contrary to dedicated systems, the design of versatile robotic systems is based on less clear requirements in terms of force, workspace, dynamics and application, making the design process less straight-forward. The presented system is advantageous in several points. It is applicable for a wide range of fields, the medical staff does not need to learn the handling of different systems and interfaces, the system makes the implementation of future surgical techniques and future hard- and software components possible, and even the adaption of third party tools is possible through the definition of an open interface.

This paper presents $M I C A$, a novel instrument for minimally invasive robotic surgery (MIRS). Following the concept of the MIRO robotic arm, which is the central part of the German Aerospace Center's (DLR) MiroSurge scenario presented in [1], the design of a versatile drive unit for various interventive tool categories is a logical consequence.

*Institute of Robotics and Mechatronics, German Aerospace Center, 82234 Wessling, Germany, e-mail: Sophie. Thielmannedlr.de.

We gratefully acknowledge the Bavarian Research Foundation (BFS) for funding the MiroSurge project. Special thanks to Paul Mittermiller. Thanks also to Holger Urbanek for the pictures.

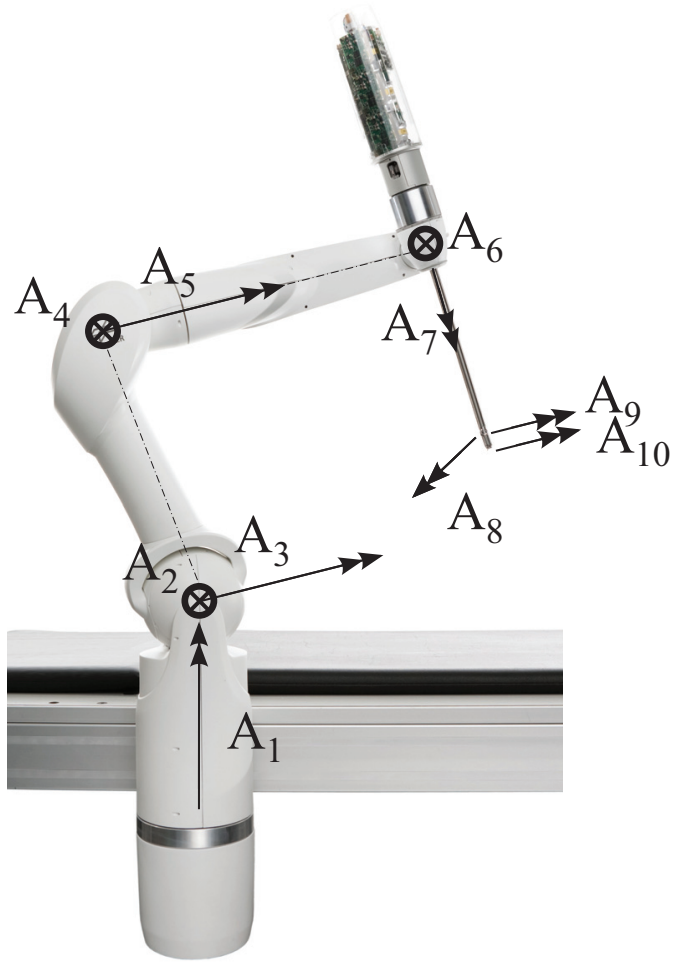

Fig. 1. MICA instrument coupled to $M I R O$ arm with joints 1-10 (1-7 for MIRO, 8-10 for MICA

The MICA instrument is a self-sufficient 3 degrees of freedom (DoFs) robot, which - in the MiroSurge system - acts as joint 8, 9 and 10 of the telemanipulator (see Fig. 1). It consists of a drive unit, a tool interface and the task specific tool with its shaft and end effector (see Fig. 2). The clear separation between the MIRO as robotic platform and the MICA as a versatile instrument with only a shared communication and power interface requires the drive unit to provide mechanical power, motor control, and sensor signal conditioning electronics. It provides the mechanical actuation for the tool by means of 3 linear motions as well as mechatronic interfaces to the robot arm and the attached tool. In the present configuration the tool is comprised of a $2 \mathrm{DoF}$ wrist, $1 \mathrm{DoF}$ gripper and $7 \mathrm{DoF}$ force/torque sensor. However, tools can range from grippers, scissors, and needle holders in MIRS to stand-alone applications for other medical fields such as NOTES (Natural Orifice Transluminal Endoscopic Surgery) since the drive unit is self sufficient and can be used both with and without the MIRO arm.

This paper will start with an introduction into the field 


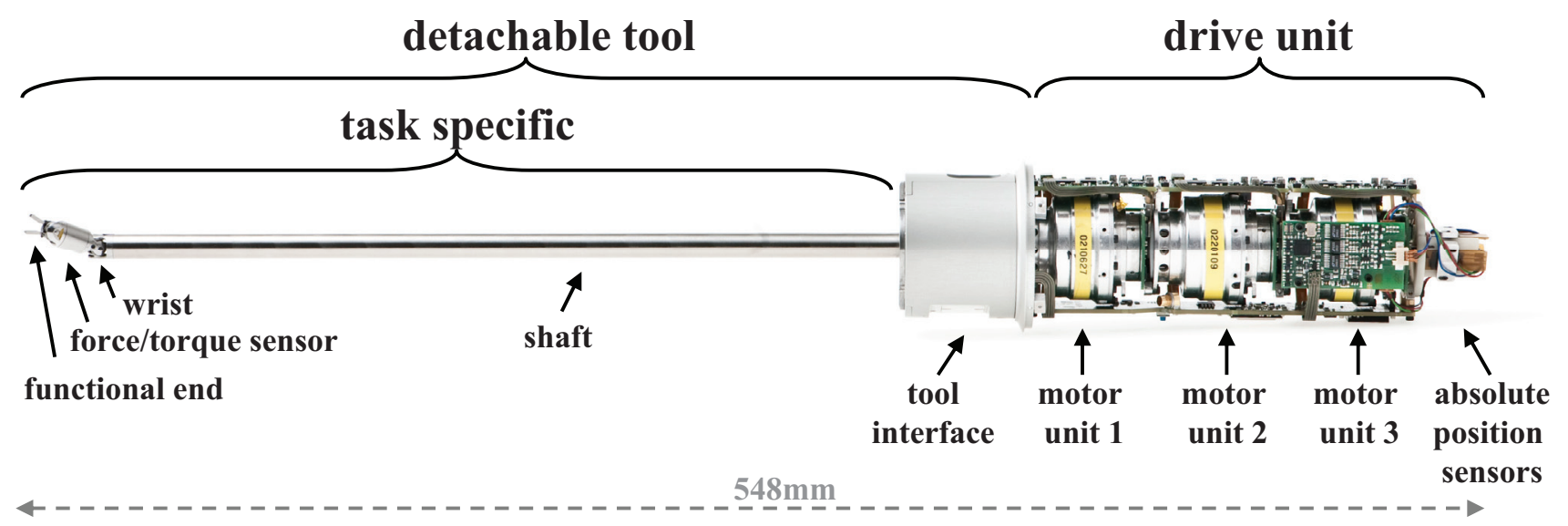

Fig. 2. Description of the MICA instrument with drive unit and tool

of related research (Sec. II), continue with a summary of the requirements which constitute the basis for the design of the MICA instrument (Sec. III). The instrument layout is described in section IV and includes the physical structure as well as the system topology with communication architecture, infrastructure, actuators, sensors, joint module and real time host. Finally, the results of some early experiments are described in section $\mathrm{V}$ and finally a conclusion and an outlook (Sec. VI) are given.

\section{RELATED RESEARCH}

The location of motors and electronics for controlling a tool for MIRS is one of the factors differentiating dedicated robotic systems from versatile designs. Dedicated systems connect to limited a set of proprietary instruments; the actuators can therefore be placed within the robotic arm. This results in lighter and less complex instruments at the expense of heavier and more complex robotic arms.

Applications within versatile systems might not always require the use of actuated tools. The integration of actuators into the robotic arm would constitute unnecessary weight and complexity in those applications. Moreover, the placement of motors in the instrument allows for more flexibility in terms of degrees of freedom as well as the integration of sensors and kinematics. Contrary, with motors and electronics in the robotic arm, the interface to the instrument is highly restricted to a specific configuration of the tools.

Providing means of detaching the surgical tool from its according drive system is required for two reasons: Firstly, the actuators are often not sterilizable, however the tool is in contact with the patient's body. Secondly, providing the surgeon with an interchangeable set of tools such as scissors and various grippers, can only be achieved in an efficient and economical manner if the drive system is versatile reusable.

The daVinci surgical system with the EndoWrist instruments by IntuitiveSurgical[2], as well as the RAVEN surgical system by the University of Washington [3] are designed as dedicated systems. All actuators and electronics are located in the robotic arms and the driving motion is transmitted mechanically to the instrument interface. The instrument therefore only consists of the coupling mechanism that transmits the motion and the tool itself, resulting in light, rugged and comparably inexpensive tools. However, the addition of any functionality not already implemented by the four actuators or the addition of sensors into the tool requires external interfaces. Such additions not only increase the complexity of the tool itself, but also complicate the switching of tools by necessitating additional connections.

The multi DoF active forceps described by Y. Kobayashi et.al. [4], the MIRS system described by J. Arata et.al. [5], the forceps manipulator described by H. Yamashita et.al. [6] and the micro manipulator described by K. Harada et.al. [7] all consist of an instrument with integrated motors and all acknowledge the necessity for detachable tools with actuated wrists. The system by Harada uses a cable driven wrist with a tool diameter of less than $3 \mathrm{~mm}$. It is designed for an openMRI environment (magnetic resonance imaging), actuated by ultrasonic motors and positioned with a passive instrument carrier. All other systems employ linear sliding linkages driven by servomotor and ball screw assemblies to actuate the tools, which are generally about $10 \mathrm{~mm}$ in diameter. The systems by Kobayashi and Arata are designed to work with an active 4 DoF remote center of motion robotic arm. While these systems follow a more versatile design approach by integrating the motors into the instrument itself, all rely on external control and drive electronics. A large number of electrical connections for motor commutation and power, position and other sensor signals is required to operate these instruments. Not only is this kind of interface complicated and specialized, it will also be susceptible to losses, noise and EMI (electromagnetic interference). Moreover, these instruments describe the use of small servo motors, in the $2.5 \mathrm{~W}$ to $11 \mathrm{~W}$ range, therefore we conclude that they are designed for manual guidance with typical hand movement frequencies limited to less than $1 \mathrm{~Hz}$ and tissue manipulation forces of few Newtons (see calculations in Sec. III).

Of all instruments presented so far, only Arata [5] shows the integration of a gripping force sensor into a modified version of the tool. Especially with the integration of force sensors the issues of signal noise and EMI become more 
prevalent, as the sensor output amplitudes can be very small (in the range of few $\mathrm{mV}$ for strain gauges). Signal conditioning and digitization should be located as close as possible to the sensor itself.

In order to provide a truly versatile instrument design, not only should the instrument contain all necessary motors, gears, control and sensor electronics, the drive unit should also provide two well structured interfaces: one towards a detachable tool and another towards the overall robotic system.

\section{REQUIREMENTS}

Foremost, the MICA is designed as an interventive instrument coupled to the $M I R O$ arm for minimally invasive procedures. Conformance to the MiroSurge setup outlines a number of requirements for the instrument design.

- Compact mechatronic interface between robot and instrument. Due to the versatile design concept of the $M I R O$, a single mechatronic interface is provided to any instrument consisting of a mechanical attachment as well as an electrical power connection and a high speed digital communication interface. Still, quick instrument changes have to be possible during surgical procedures.

- Conformance to the MiroSurge system topology (see Sec. IV-B)

- Lightweight design. Added weight at the TCP will adversely effect the dynamic performance of the robotic arm.

- Compact design of the drive unit, particularly in diameter. Multiple robotic arms will be working in close proximity around the operating table. Interference and collisions of instruments have to be avoided.

Various tools targeted at different surgical applications will be used with the MICA drive train, differing in the number of DoFs, functionality of the end effector, and range of motion. Aside from the interface between robot and instrument a second mechatronic interface between drive train and tool is implemented. This interface provides suitable mechanical transmission to the tool as well as standardized digital communication with any sensors located in the tool, such as the DLR force/torque sensor described in [8].

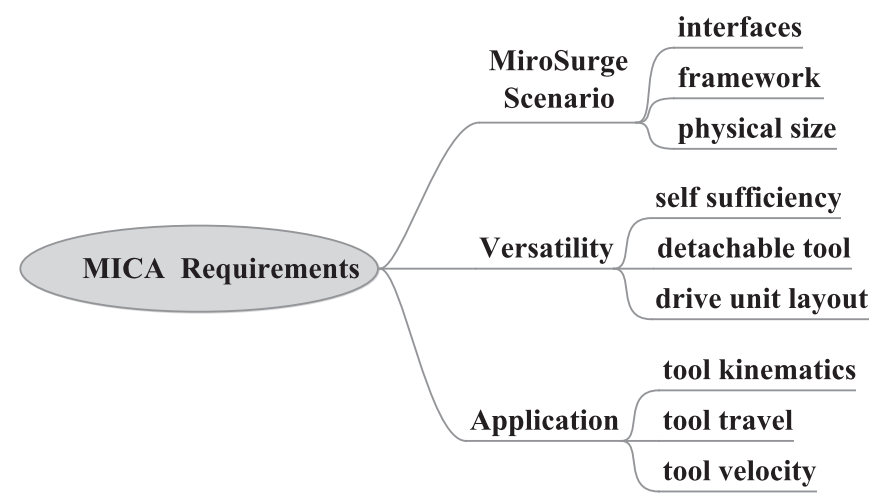

Fig. 3. Requirements for the design of the MICA instrument

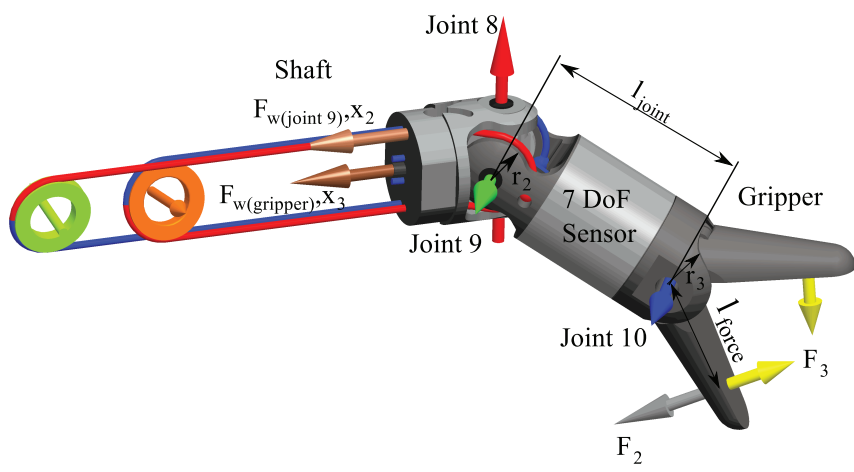

Fig. 4. Forces and link-lengths of the the cable-driven articulated gripper presented in [8]. Joint 8 is perpendicular to joint $9 . F_{w(j o i n t 8)}$ not shown.

TABLE I

SPECIFICATIONS OF THE CURRENT END EFFECTOR AND REQUIRED FORCES

\begin{tabular}{llllll}
\hline Distance & {$[\mathrm{mm}]$} & Angle & {$[\mathrm{rad}]$} & Force & {$[\mathrm{N}]$} \\
\hline$l_{\text {force }}$ & 9.65 & $q_{8}$ & \pm 0.7 & $F_{2}$ & 10.00 \\
$l_{\text {joint }}$ & 18.82 & $q_{9}$ & \pm 0.7 & $F_{3}$ & 10.00 \\
$r_{2}$ & 2.95 & $q_{10}$ & $0-1.22$ & & \\
$r_{3}$ & 3.25 & & & & \\
\hline
\end{tabular}

MIRS at the beating heart is the most demanding application within the MiroSurge scenario in terms of tool dynamics. Using frequency analysis, Ortmaier [9] found the dominant frequencies of the non-sinusoidal stabilized motion of the heart surface to be in the range of $0.24 \mathrm{~Hz}, 1.2 \mathrm{~Hz}, 2.4 \mathrm{~Hz}$, and $3.5 \mathrm{~Hz}$.

For the dimensioning of the drive unit, a motion frequency of $5 \mathrm{~Hz}$ and tool kinematics based on the $3 \mathrm{DoF}$ articulated gripper for MIRS (see Tab. III and Fig. 4) are assumed. In addition, performance should leave ample room for expansion.

With the assumed forces $F_{2,3}$ of $10 \mathrm{~N}$ inside of the branches for gripping and manipulation of tissue (see Tab. I and Fig. 4) the forces in the drive cables can be computed $\left(F_{w(\text { joint } 8)}=F_{w(\text { joint } 9)}\right)$ :

$$
\begin{aligned}
& F_{w(\text { joint } 9)_{\max }}=\frac{F_{2}}{r_{2}} \cdot\left(l_{\text {joint }}+l_{\text {force }}\right)=96.51 \mathrm{~N} \\
& F_{w(\text { gripper })}=2 \cdot \frac{F_{3}}{r_{3}} \cdot l_{\text {force }} \quad=59.38 \mathrm{~N}
\end{aligned}
$$

Aside from forces, the stroke length $x_{i}$ is fundamental for the design of the drive train. As joint 8 and 9 are coupled, strokes for the two drive cables $x_{1}$ (blue) and $x_{2}$ (red in Fig. 4) are calculated as follows:

$$
\left(\begin{array}{l}
x_{1} \\
x_{2}
\end{array}\right)=\left(\begin{array}{cc}
1 & -1 \\
1 & 1
\end{array}\right) \cdot\left(\begin{array}{l}
q_{8} \\
q_{9}
\end{array}\right) \cdot r_{2}
$$

Hence, the maximum for $x_{1}$ and $x_{2}$ is at the maximal deflection of the joints 8 and 9: $x_{1}=x_{2}= \pm 4.13 \mathrm{~mm}$.

The gripper is actuated by a third cable, which is split at the end effector (not shown in Fig. 4). The stroke length is 
TABLE II

REQUIREMENTS FOR THE DESIGN

\begin{tabular}{llllll}
\hline Strokes & {$[\mathrm{mm}]$} & Forces & {$[\mathrm{N}]$} & Param. & Value \\
\hline$x_{1}$ & 8.26 & $F_{w(j 8)}$ & 96.51 & $a_{\min }$ & $1980 \mathrm{~mm} / \mathrm{s} 2$ \\
$x_{2}$ & 8.26 & $F_{w(j 9)}$ & 96.51 & $v_{\max }$ & $99 \mathrm{~mm} / \mathrm{s}$ \\
$x_{3}$ & 3.97 & $F_{w(g r)}$ & 59.38 & $M_{\text {mot }}$ & $0.130 \mathrm{Nm}$ \\
& & & & $P_{\text {mot }}$ & $40.75 \mathrm{~W}$ \\
\hline
\end{tabular}

dependent on $r_{3}$ and the range of motion $q_{10}$ :

$$
x_{3}=q_{10} \cdot r_{3}=3.97 \mathrm{~mm}
$$

The motion frequency of $5 \mathrm{~Hz}$ and the calculated actuation length of cables 1 and 2 lead to the minimal needed acceleration of the linear actuator with $1980 \mathrm{~mm} / \mathrm{s}^{2}$, yielding a maximum speed of $99 \mathrm{~mm} / \mathrm{s}$.

A ball screw with a pitch of $2 \mathrm{~mm}$ and a diameter of $12 \mathrm{~mm}$ is used in the drive train as shown in Fig. 5. With the inertia of the power train, a manipulation force of $10 \mathrm{~N}$ at the end effector and an acceleration of $a_{\text {min }}$, the driving torque of one motor can be computed. With $M=J * \dot{\omega}$ and $J_{\text {total }}=J_{\text {rotor }}+J_{\text {nut }}+J_{\text {spindle }}+J_{\text {force }}$ and $\omega_{\max }=311 \mathrm{rad} / \mathrm{s}$ and $\dot{\omega}_{\min }=6220.3 \mathrm{rad} / \mathrm{s}^{2}$ follows:

$$
\begin{aligned}
M_{\text {motor }} & =0.130 \mathrm{Nm} \\
P_{\text {motor }} & =M_{\text {motor }} \cdot \frac{v_{\text {max }} \cdot 2 \pi}{\text { pitch }}=40.75 \mathrm{~W}
\end{aligned}
$$

Tab. II sums up the requirements for the design of an instrument for the MiroSurge scenario.

\section{FUNCTIONAL DESIGN}

This section gives an overview of the mechanical implementation and physical principles, continues with the system topology and infrastructure and concludes with a description of the major components: actuators, sensors, tool interface, joint module and real time host.

\section{A. Physical structure}

Fig. 2 illustrates the layout of the MICA instrument. The versatile drive unit (on the right side) provides actuation, position sensing, signal processing and communication. The application specific tool (on the left side) transfers the forces and motions to the operation site, senses forces and torques applied to the end effector and transmits these signals to the tool interface.

The drive train consists of:

- 3 motor units with ball screws

- 3 input-side incremental position sensors (situated in motor units for rotational motor position) and 3 outputside absolute position sensors (situated at the right end of the drive train for linear ball screw position)

- electronic components

- interface to tool (mechanical and electrical) and to robot (electrical)

Whereas the detachable tool consists of:

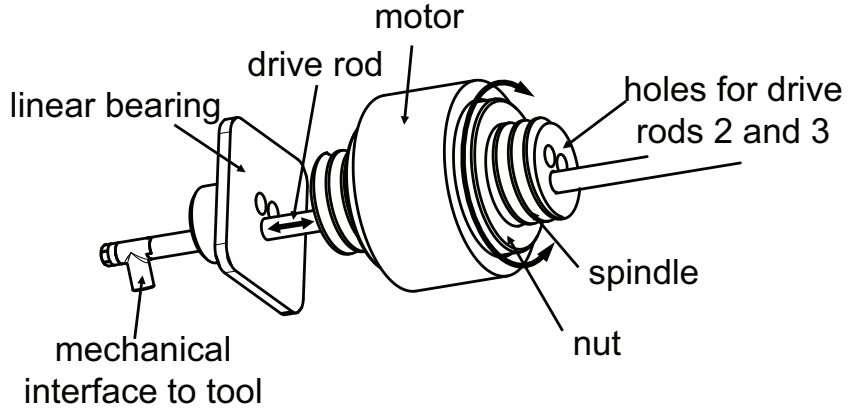

Fig. 5. Functional design of a motor unit

- 'tool interface' with mechatronic connection to the drive unit and mechanical coupling of entire instrument to the robot

- shaft

- 2 DoF wrist with 6+1 DoF force/torque sensor

- functional end

The drive unit provides any coupled tool with a mechanical interface with three linearly moving drive rods. To actuate these rods, the motor units are designed as in Fig. 5. A rotatory actuator (motor) drives the nut of a ball screw. As the linear bearing and the excentric mounting of the drive rod prevent the rotation of the spindle, it moves linearly. Two holes in the spindle allow a concentric arrangement of the 3 motor units rotated by $120^{\circ}$. Thus, for each motor unit the drive rods of the two remaining motor units are passed through the spindle of the first.

At the tool-sided end of the drive rods, connection elements are mounted perpendicularly in order to transmit push-pull actuation through the detachable mechanical tool interface.

Even though the drive train provides three linear actuated rods as mechanical interface, tendon-driven tools can also be coupled. In this case, a specific tool interface transforms the motion of the drive rods into a movement of cables. The tool interface also provides the mechanical coupling of the whole instrument to the robot via a magnetic clutch located at the robot and enables quick tool changes during clinical use.

The drive unit is located above the MIRO wrist for safety reasons. In the event of complete system- or power failure manual instrument removal is possible by extracting the instrument upwards along the line of the trocar. In the case of mechanical overload of the instrument, the coupling will release as safety feature, however, the instrument will not drop towards the patient. As rotation of the tool shaft is provided by the MIRO wrist, a coaxial arrangement of tool, robot wrist and drive unit is necessary. Due to this arrangement, tool changes during a surgical procedure involve removing the entire instrument.

The tool interface combines several functional components:

- A system specific interface to the drive unit. This interface mechanically and electrically connects the tool 


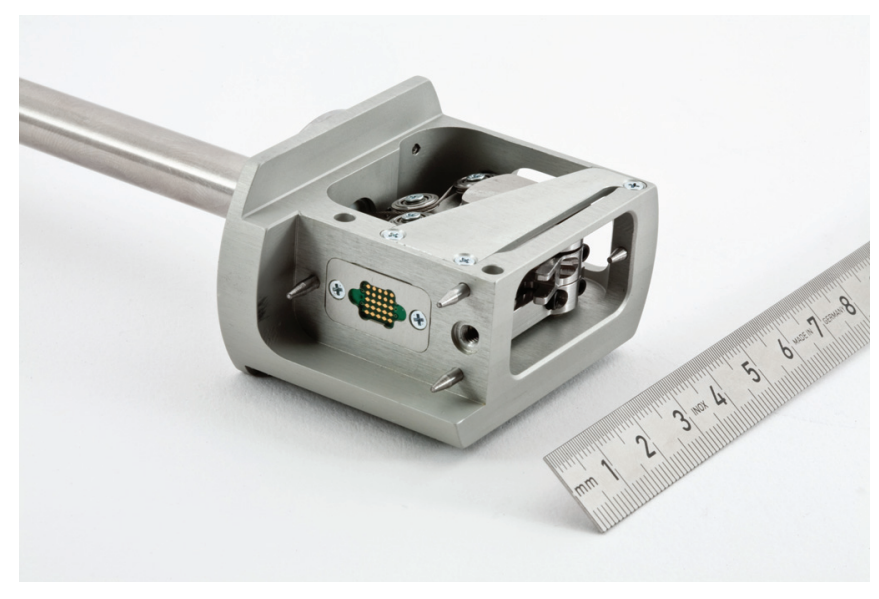

Fig. 6. Tool interface

to the drive unit creating the instrument. A tongue-andgroove-like connection is established with the actuator rods of the drive unit. Careful design of the mating surface geometry, allows connection even if the mating surfaces are initially misaligned (see Fig. 7).

- A system specific interface to the MIRO arm. This interface provides secure, switchable magnetic attachment of the MICA to the MIRO This is the location of the sterile barrier between $M I R O$ and MICA.

- A tool specific linkage or pulley system translating the linear motion of the drive unit's actuator rods to the motion required for articulating the tool.

\section{B. System topology}

The requirement to integrate different tools with the $M I C A$ drive train calls for a modular, open system architecture. In spite of this flexibility, the requirement of high integration necessitates dedicated, efficient implementation of components. The availability of independently tested components enables faster development cycles for different tool configurations.

To balance these opposing requirements of flexibility and high integration the MICA platform is designed as a layered component architecture, starting at dedicated, highly integrated components leading towards general-purpose, commercial off-the shelf platforms (see Fig. 7).

The sensor/actuator modules constitute the most dedicated level of the architecture as they represent the interface to the physical world. These mechatronic components comprise an electronic design that is tightly integrated with the mechanical design and the associated software. All signals are digitized at this level. Thus, signal noise, cabling effort, and EMI issues are reduced drastically.

At the next level the joint module provides the infrastructure for the integration of various sensor and actuator modules. Hence, the key for an open platform is the flexibility of the joint module. At this granularity, Field Programmable Gate Array (FPGA) technology is a suitable means for providing the desired flexibility at reasonable design costs. For example, the great number of available $\mathrm{I} / \mathrm{O}$ pins of the FPGA allows the parallel connection of all sensor/actuator modules. Thus, low bandwidth communication protocols featuring small implementation footprints can be used here.

At top level, a real-time computing host provides the necessary computing power for the control algorithms of a control application.

The applied communication protocols follow the same hierarchy as the sensor and actuator modules: Close to hardware, low bandwidth protocols featuring a small implementation footprint are used, while the general-purpose platforms are connected by high-bandwidth standard communication protocols

The design of the components and of their supporting infrastructure both are governed by the domain model "The Virtual Path" that has been developed especially for the design of the MiroSurge system [10]. "The Virtual Path" constitutes an abstract infrastructure specification as it defines synchronization, scheduling, and error handling of the system components.

1) Communication Architecture: Communications connecting the sensor/actuator modules require a small implementation footprint since it is targeted at FPGA and CPLD platforms. The industry standard sensor bus BiSS C-Mode is a reliable bidirectional bus which is capable of transferring desired and actual data in the same cycle [11]. It is an open, license-free serial bus with data rates up to $10 \mathrm{Mbit} / \mathrm{s}$. Cascading of up to 8 devices is feasible. The protocol overhead is small and the bus uses three wires only; however, data transfer is reliable since a cyclic redundancy check is included.

The joint module is connected to the real-time computing host by the same communication that is used in the MIRO robot: SpaceWire is a packet-based bus that is deterministic for a given topology [12]. Thus, the MICA instrument can be directly connected to the MIRO wrist in order to avoid additional cabling. The physical layer is implemented with an IEEE 802.3 Gigabit Ethernet compliant ethernet transceiver featuring $1 \mathrm{Gbit} / \mathrm{sec}$ bandwidth and a typical latency lower than $20 \mu \mathrm{s}$.

2) Infrastructure: The infrastructure of a mechatronic system enables the integration of the distributed electronic hardware, such as sensors and actuators, with control applications. Therefore, it has to provide convenient interfaces for application developers and constitutes an efficient but transparent adhesion between the distributed components. Thus, infrastructure involves the operational environment for sensors and actuators, communication protocols and software drivers. The MICA infrastructure implements a Hardware Abstraction Layer (HAL) as the convenient interface and a middleware for the communication implementation. As mentioned above, the infrastructure design for the MICA is governed by the "The Virtual Path" domain model that defines synchronization, scheduling, and error handling for all system components.

"The Virtual Path" model is based on the formal Synchronous Model, that assumes discrete signals with a fixed 
sample period [13]. While the classical system view is rather software-centric, "The Virtual Path" model is radically different: The entire system is synchronized by its sensors. The high-level software implementations of control algorithms are triggered by the hardware components and the software is scheduled on demand, i.e. when new data is available.

In conformance to the discrete signal model the distributed component functionality is transparently integrated by a signal-flow-oriented middleware specifically developed for this purpose. Utilizing a static system specification approach and compile time optimization, the typical overhead of common middleware implementations is avoided and a small run-time footprint is achieved [14].

The HAL is the functional separation between instrument hardware and instrument control. For convenience, the HAL implementation provides a complete current controlled instrument to the control designers and presents all sensor values as floating point SI-values. Since Cartesian control and joint control are implemented with MATLAB Simulink, the HAL is represented as a Simulink block. The HAL not only integrates the remote joint functionality. It implements also all those features that are not provided by the hardware but which are necessary for a suitable view of a current controlled instrument, such as sensor value calibration.

3) Actuators: One motor module is a compact selfcontained intelligent component and consists of a DLRdesigned permanent-magnet synchronous machine (PMSM), power electronics and an input-sided rotational position sensor as depicted in Fig. 8.

The PMSM is especially designed for robotic applications (mainly high torque and low rpm) and optimized for low losses. This brushless direct current (BLDC) motor features a hollow shaft with an open inside diameter of $18 \mathrm{~mm}$. This makes it ideally suited for coaxially mounting a ball screw directly inside the rotor as described in Sec. IV-A.

The position sensor is a magneto-resistive sensor with a resolution of $10752 \frac{\text { Increments }}{\text { revolution }}$ connected via BiSS Interface. Sensor data is used for commutation of the motor as well as
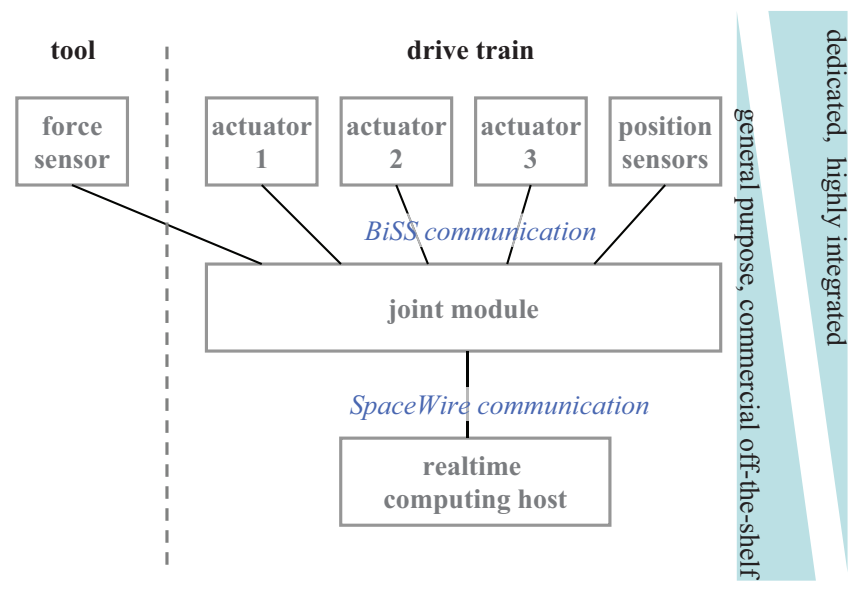

Fig. 7. System topology

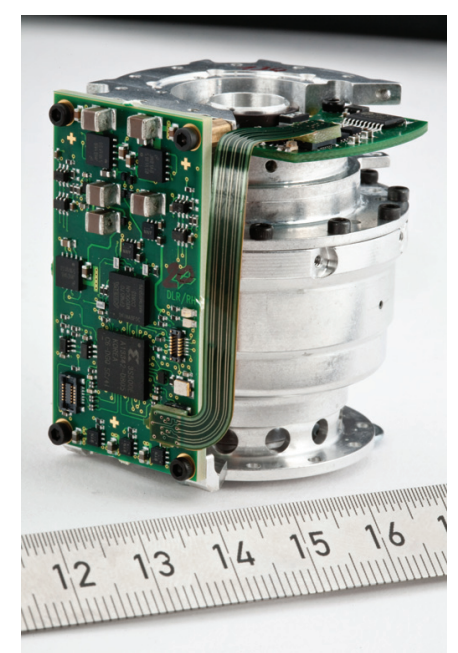

Fig. 8. One of three motor modules (metric scale ruler)

position source for the superimposed controllers.

The power electronics is mounted tangentially to the motor in order to keep power and signal connections as short as possible to reduce EMI issues. The electronics consists of the motor drivers as well as signal processing elements. Signal processing is accomplished on a Xilinx Spartan3e XC3S500EP132 with a footprint of only $8 \times 8 \mathrm{~mm}$. This FPGA is tasked with both, communication (BiSS) with the joint electronics and position sensor, as well as current control of the motor. The current of the PMSM is controlled in a state-of-the-art field-oriented approach with 2 PI controllers, decoupling network, feed forward path and space vector modulation [15]. This control approach enables fast settlement of the desired currents, hence superimposed controllers can treat the motor module as an ideal torque source. The control period is chosen to be $100 \mathrm{kHz}$. This high sampling rate is due to low electrical constants and high dynamics of the motor as well as the need for low DC link ripples and small filter dimensions. Furthermore, the power electronics implements safety (i.e. single point of failure (SPOF) detection, actuator state machine [10]) and housekeeping functions. In order to detect and switch off motor power at any SPOF the power electronics utilizes an additional Altera MAX2 CPLD with own power supply. The Spartan3e and the MAX2 check each other's state; in case of failure, a safe torque-off strategy is introduced in order to prevent physical interaction of the motor.

The result is a very compact and self sufficient motor module as depicted in Fig. 8.

4) Sensors: As a consequence of the above described system topology, sensor data is amplified and digitized directly at the sensor's location. The communication interface with BiSS protocol, CRC check and data acquisition timing is realized in a MAX 2 CPLD at the same location. This principle holds for the output-sided absolute position sensors measuring the three rod positions by linear potentiometers as well as the force sensor in the 
tool measuring interaction forces, torques and the gripping force directly at the tool tip. The previously described motor-sided incremental position sensor implements the same communication protocol.

5) Joint Module: The joint Module comprises 3 tasks:

- signal aggregation

- routing and conversion

- interpretation

The aggregation of the actual data is triggered by the $3 \mathrm{kHz}$ main control loop. If the joint module recognizes a new cycle, it immediately requests actual data of the actuators and sensors by initiating a data exchange. Afterwards the collected data is sent to the control application to calculate the desired control input. Reception of the calculated desired data initiates an additional data exchange which provides the actuators and sensors with the new desired data. Data exchange happens by using the routing and conversion feature of the joint module.

SpaceWire packets are received from the control application and routed into the appropriate protocol (BiSS, SPI, I2C) to the actuator or sensor target and vice versa. Data is also converted between the different data representations used by the control application and the actuator or sensor.

Exchanged data is also interpreted concerning plausibility. An overflow, a communication error or the absence of actual data cause the particular data set of an actuator or sensor to be marked with the additional error information and the control application can react accordingly.

6) Real Time Host: Following the strategy to implement as much functionality as possible on general-purpose architectures all control algorithms besides motor current control are implemented on the external controller host, which is a standard PC platform running the real-time OS QNX [16]. This approach allows for the use of conventional development tools such as Mathwork's MATLAB Simulink and enables efficient algorithm and application development. MICA's HAL provides the interface to the development tools. Moreover, the user benefits directly from the constantly rising computing power of Commercial of the Shelf (COTS) platforms.

\section{RESULTS}

To verify that the MICA satisfies the requirements postulated in Sec. III, (overall specs of the MICA are shown in Tab. III), initial experiments were conducted, commanding a step and sinusoidal trajectory for each motor module. Emphasis during these experiments was placed purely on actuator dynamic response, not on controller design. Therefore, a simple P-controller was used.

Fig. 9 shows the behavior of the motor in response to a step input trajectory: On the left side the actual motor position converted into rod position (red) is compared to the desired position input (black). On the right side the actual rod velocity (red) is compared to the desired input velocity (black), which is infinite for a step position input.
TABLE III

SPECIFICATIONS OF THE MICA INSTRUMENT

\begin{tabular}{ll}
\hline Specifications & Value \\
\hline Number of joints & 2 (wrist) +1 (functional end) \\
Mass of drive unit & $695 \mathrm{~g}$ \\
Mass of entire instrument & $838 \mathrm{~g}$ \\
Length of drive train & $176 \mathrm{~mm}$ \\
Length of tool interface & $41.9 \mathrm{~mm}$ \\
Length of shaft & $300.0 \mathrm{~mm}$ \\
Length of entire instrument & $548 \mathrm{~mm}$ \\
Joint length & $18.8 \mathrm{~mm}$ \\
Position sensors & $6(3 \mathrm{motor}-$ sided; 3 output-sided) \\
Force/Torque Sensor & $1(6+1$ DoF, endeffector-sided) \\
Control cycle & $3 \mathrm{kHz}$ \\
latency & $<200 \mu \mathrm{s}$ \\
\hline Max. rod velocity & $120 \mathrm{~mm} / \mathrm{s}$ \\
\hline
\end{tabular}
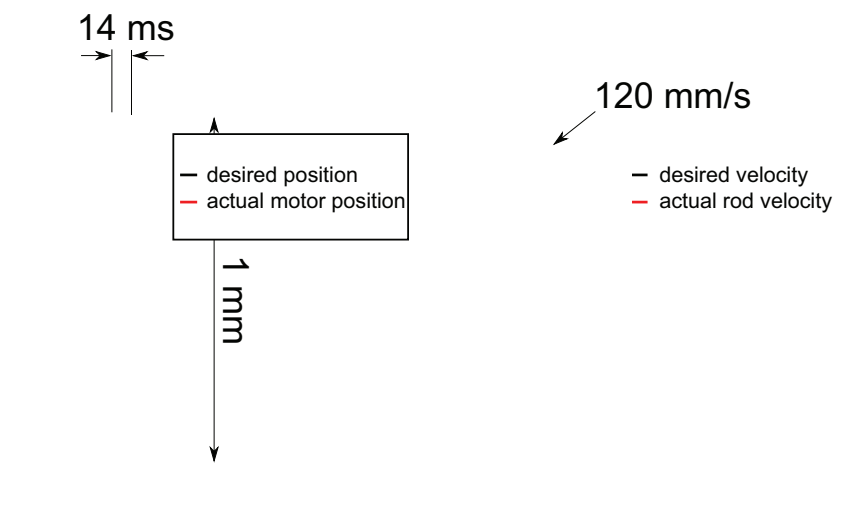

Fig. 9. Step response of one motor unit at a current limit of 9 A. On the left side positions are compared, on the right side, the velocity of the rod is measured

With the motor current limited to 9 Amperes a step of $1 \mathrm{~mm}$ is commanded (this equals $17.4 \mathrm{deg}$ end effector articulation). The motor accelerates within $14 \mathrm{~ms}$ to a maximum velocity of $120 \mathrm{~mm} / \mathrm{s}$ (graph on the right side). This equals an acceleration of $8571 \mathrm{~mm} / \mathrm{s}^{2}$. Controller overshoot and oscillation are clearly visible and should be optimized in the future by finding suitable controller parameters.

A second experiment was conducted to evaluate the instrument response to a fast sinusoidal trajectory with medium amplitude. Fig. 11 shows the desired position (black) with an amplitude of $\pm 2 \mathrm{~mm}$ and a frequency of $7.5 \mathrm{~Hz}$ compared to the actual motor position (blue) and rod positions (red).

A slight delay between desired and actual motor position is visible. The error rate is not plotted separately as this experiment was conducted to show the dynamics of the mechatronic system rather than the quality of the control scheme. Obviuosly, using a simple P-controller will introduce a certain amount of delay and prevent the actual position from reaching the desired position. These errors will grow with actuation frequency. Hysteresis of about $0.1 \mathrm{~mm}$ exists between actual motor position and actual rod position signals. This is attributed to an inaccuracy in the linear potentiometer measuring the rod position. It also has to be 


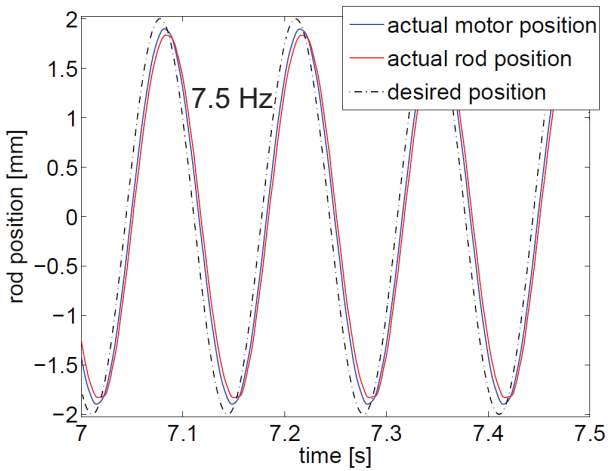

Fig. 10. Experimental data for sinusoid movement of rod with an amplitude of $2 \mathrm{~mm}$ and a frequency of $7.5 \mathrm{~Hz}$

noted that the current tool design does not include position sensors in the functional end. Therefore, errors stemming from deflection of the shaft under load and elastic effects in the drive cable cannot be measured directly. However, interaction forces responsible for these elastic deformations are measured by the $7 \mathrm{DoF}$ force/torque sensor at the tool tip and can be included in the control scheme.

\section{CONCLUSION/OUTLOOK}

This paper presents a novel drive unit for minimally invasive surgery applications. It is self-sufficient in terms of electronics and motor control, provides ample dynamics for surgery at the beating heart and offers two simple interfaces for the robot and for 3 DoF tools. Clearly defined topology and mechatronic interfaces enable the development of tools by other groups.

Early experiments and results presented in Sec. V show that the MICA drive unit meets the requirements described in Sec. III. However, further experiments will be needed to analyze the concept in more detail.

Medical robotics could - in the future - be applied in operation theaters both more in depth (with more functionalities in one specific application: gripping, rinsing, ultrasound, cautery) as well as with increased diversity (in various application areas: telesurgery, laser cutting, biopsy, osteotomy). The MiroSurge scenario of the DLR meets these needs with the versatile robot $M I R O$ geared towards more diverse application areas and the versatile instrument $M I C A$ as building block for instruments with increased functionality.

\section{REFERENCES}

[1] Ulrich Hagn, R. Konietschke, A. Tobergte, M. Nickl, S. Jörg, B. Kübler, G. Passig, M. Gröger, F. Fröhlich, U. Seibold, L. LeTien, A. Albu-Schäffer, A. Nothhelfer, F. Hacker, M. Grebenstein, and G. Hirzinger. DLR MiroSurge: a versatile system for research in endoscopic telesurgery. International Journal of Computer Assisted Radiology and Surgery, 2009.

[2] G. Guthart and J. Salisbury. The intuitive telesurgery system: Overview and application. In Procedings of the IEEE International Conference on Robotics and Automation (ICRA), volume 1, pages 618-621, San Francisco, CA, USA, April 2000. ISBN 0-7803-5886-4.

[3] Jacob Rosen and Blake Hannaford. Doc at a distance. IEEE Spectrum, pages 34-39, October 2006.

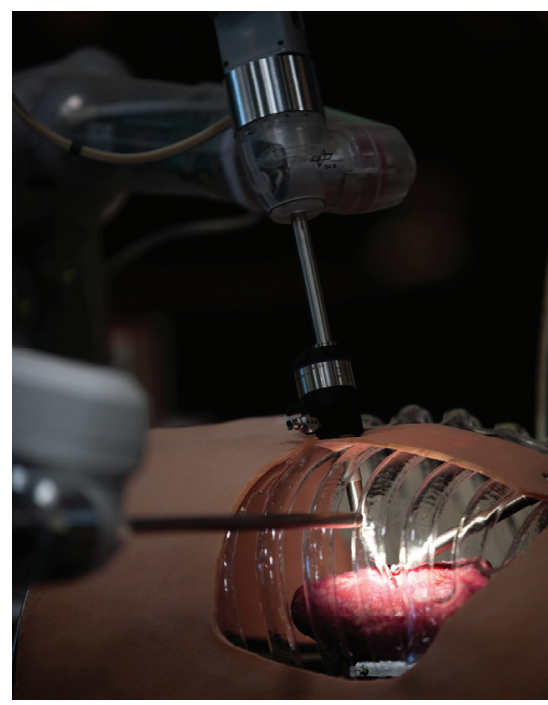

Fig. 11. The MiroSurge system in the teleoperation setup manipulating on the DLR beating heart simulator

[4] Yuki Kobayashi, Shingo Chiyoda, Kouichi Watabea, Masafumi Okada, and Yoshihiko Nakamura. Small occupancy robotic mechanisms for endoscopic surgery. In Takeyoshi Dohi and Ron Kikins, editors, $M I C$ CAI - Medical Image Computing and Computer-Assisted Intervention, volume 2488 of Lecture Notes in Computer Science, pages 75-82. Springer, September 2002.

[5] Jumpei Arata, Mamoru Mitsuishi, Shinichi Warisawa, Katsuya Tanaka, Takashi Yoshizawa, and Makoto Hashizume. Development of a dexterous minimally-invasive surgical system with augmented force feedback capability. In 2005 IEEE/RSJ International Conference on Intelligent Robots and Systems, 2005.

[6] H. Yamashita, A. Iimura, E. Aoki, T. Suzuki, T. Nakazawa, E. Kobayashi, M. Hashizume, I. Sakuma, and T. Dohi. Development of endoscopic forceps manipulator using multi-slider linkage mechanisms. In Proceeding of the 1st Asian Symposium on Computer Aided Surgery - Robotic and Image guided Surgery, Ibaraki, Japan, April 2005

[7] Kanako Harada, Kota Tsubouchi, Masakatsu G. Fujie, and Toshio Chiba. Micro manipulators for intrauterine fetal surgery in an open mri. In Proceedings of the IEEE International Conference on Robotics and Automation (ICRA), pages 502- 507, 2005.

[8] U. Seibold, B. Kuebler, and G. Hirzinger. Medical Robotics, chapter Prototypic force feedback instrument for minimally invasive robotic surgery, pages 377-400. I-Tech Education and Publishing, Vienna, Austria, 2008. ISBN-13: 978-3-902613-18-9.

[9] T. Ortmaier, M. Gröger, D.H. Boehm, V. Falk, and G. Hirzinger Motion estimation in beating heart surgery. IEEE Transactions on Biomedical Engineering (TBME), 52(10):1729-1740, October 2005.

[10] M. Nickl, S. Jörg, and G. Hirzinger. The virtual path: The domain model for the design of the miro surgical robotic system. IFAC, http://www.ifac-papersonline.net/, 2009.

[11] IC Haus, http://www.ichaus.com. BiSS Interface Protocol Description (C-Mode), c1 edition, 2007.

[12] European Cooperation for Space Standardization (ECSS), http://spacewire.esa.int. ECSS E-50-12A SpaceWire - Links, nodes, routers and networks, 2003.

[13] Albert Benveniste and Gèrard Berry. The synchronous approach to reactive and real-time systems. $P$-IEEE, 79(9):1270 - 1282, September 2001.

[14] S. Jörg, M. Nickl, and G. Hirzinger. Flexible signal-oriented hardware abstraction for rapid prototyping of robotic systems. In IROS 2006, pages 3755 - 3760, Peking, October 2006.

[15] N.P. Quang and J.A. Dittrich. Vector Control of Three-Phase AC Machines. Springer, 2008.

[16] www.qnx.com. Qnx software systems. 